Der Schnittpunkt der Curven, Fig. II, liegt also bei $+36^{0}$, bei welcher Temperatur der Uebergang des Tetrahydrats in das Monohydrat erfolgt.

\title{
Cadmi umjodid.
}

Cadmiumjodid ist nur wasserfrei bekannt; die gesättigte Lösung enthält:

\begin{tabular}{r|c|c}
\hline \hline bei $\mathrm{t}$ & $\begin{array}{c}\mathrm{g} \mathrm{Cd} \mathrm{J}_{2} \\
\text { in } 100 \mathrm{~g} \text { Lösung }\end{array}$ & $\begin{array}{c}\text { Mol. Wasser } \\
\text { auf } 1 \text { Mol. Cd J }\end{array}$ \\
\hline $0^{0}$ & 44.39 & 25.41 \\
$+18^{0}$ & 46.02 & 23.85 \\
$+50^{\circ}$ & 49.35 & 20.87 \\
$+75^{0}$ & 52.65 & 18.29 \\
$+100^{\circ}$ & 56.68 & 15.92
\end{tabular}

Diese Zahlenwerthe stimmen annähernd mit denjenigen früherer Beobachter überein. Man sieht aus der Fig. II, dass die Curve dem Schmelzpunkt des Salzes zustrebt.

Charlottenburg, 20. December 1898.

17. R. Funk: Die Löslichkeit einiger Metallnitrate. Studien über die Löslichkeit der Salze. III.

[Mittheilung aus der phys.-techn. Reichsanstalt; vorgetragen in der Sitzung rom 12. December 1898.]

Ueber die Nitrate von Magnesium, Zink, Mangan, Eisen, Kobalt, Nickel, Kupfer und Cadmium liegen zwar in der Literatur bereits zahlreiche Angaben vor, doch ist die Kenntuiss der Hydrate dieser Salze noch in vieler Beziehung lückenhaft, und namentlich sind die Löslichkeitsverhältnisse derselben noch ungenügend erforscht. Die folgende Untersuchung sucht die Curven für die Löslichkeit der einzelnen Salzhydrate festzustellen.

I. Magnesiumnitrat.

Das Magnesiumnitrat ist bisher nur in seiner Form als Hexahydrat bekannt; dasselbe schmilzt bei $90^{\circ}$ und giebt beim Versucb, 
oberhalb dieser Temperatur durch langsames Eindunsten zu einer wasserärmeren Modification zu gelangen, nach einigen Tagen einen Brei, welcher bereits schwer lösliches, basisches Salz enthält; es ist also von einem bestimmten Concentrationsgrade an ein weiteres Eindampfen der Lösung mit Verlust an Salpetersäure verbunden. Die von Ditte bescbriebenen wasserärmeren Modificationen mit 3 und 2 Mol. Wasser habe ich nicht erhalten können.

Wohl aber kann man durch Abküblen einer Lösung des Magnesiumnitrates auf $-20^{\circ}$ ein wasserreicheres Hydrat mit $9 \mathrm{Mol}$. Wasser herstellen. Dasselbe geht bereits bei $-17^{\circ}$ wieder in das Hexabydrat über.

Die Löslichkeitsbestimmungen der beiden Modificationen ergaben folgende Daten:

\begin{tabular}{|c|c|c|c|}
\hline HJdrat & Temperatur & $\begin{array}{c}\text { Procente } \\
\text { der Lösung an } \\
\mathrm{Mg}\left(\mathrm{NO}_{3}\right)_{2}\end{array}$ & $\begin{array}{l}\text { Moleküle Wasser } \\
1 \mathrm{Mol} . \stackrel{\mathrm{auf}}{\mathrm{Mg}\left(\mathrm{NO}_{3}\right)_{2}}\end{array}$ \\
\hline \multirow[t]{3}{*}{$\mathrm{Mg}\left(\mathrm{NO}_{3}\right)_{2}+9 \mathrm{H}_{2} \mathrm{O}$} & $-23^{\circ}$ & 35.44 & 15.02 \\
\hline & $-20.5^{0}$ & 36.19 & 14.50 \\
\hline & $-18^{\circ}$ & 38.03 & 13.43 \\
\hline \multirow[t]{10}{*}{$\mathrm{Mg}\left(\mathrm{NO}_{3 / 2}\right)+6 \mathrm{H}_{2} \mathrm{O}$} & $-18^{\circ}$ & 38.03 & 13.43 \\
\hline & $-4.5^{\circ}$ & 39.50 & 12.63 \\
\hline & $0^{0}$ & 39.96 & 12.38 \\
\hline & $+18^{\circ}$ & 42.33 & 11.23 \\
\hline & $+40^{\circ}$ & $4.5 .8 i$ & 9.73 Schmp. \\
\hline & $+80^{\circ}$ & $5: 3.6 ! 1$ & 7.11 \\
\hline & $+90^{\circ}$ & 57.81 & 6.00 \\
\hline & $+89^{0}$ & 133.14 & 4.81 \\
\hline & $+77.5^{\circ}$ & 6.67 & 4.31 \\
\hline & $+1 i 7^{\circ}$ & 67.55 & 3.95 \\
\hline
\end{tabular}

Die Curve des Hexahydrates hat, wie aus der Tabelle und der Curvenzeichnung zu ersehen, einen rückläufigen Ast, d. h. das Salz ist im Stande, gesättigte Lösungen zu bilden, welche weniger Wasser enthalten, als das an Boden liegende Hexabydrat. und welche man als Lösungen des letzteren in der wasserfreien Substanz auffassen kann. Derartige Lösungen stellt man ber, indem man das Hexahydrat über Schwefelsăure entwässert und das entwässerte Product mit mehr oder weniger Krystallen des Hexahydrates zusammenschmilzt. Beim Abküblen der Schmelze scheiden sicb dann Krystalle des Hexahydrates aus, welche sich bei geringer Temperaturerhöhung wieder auflösen. 
Zur Vervollständigung des Curvenbildes wurde noch die Gefrierpanktscurve ermittelt. Dies geschab in der Weise, dass Lösungers von bekanntem Gehalt durch $A b k u ̈ h l e n$ und Rühren mit einem Glas-

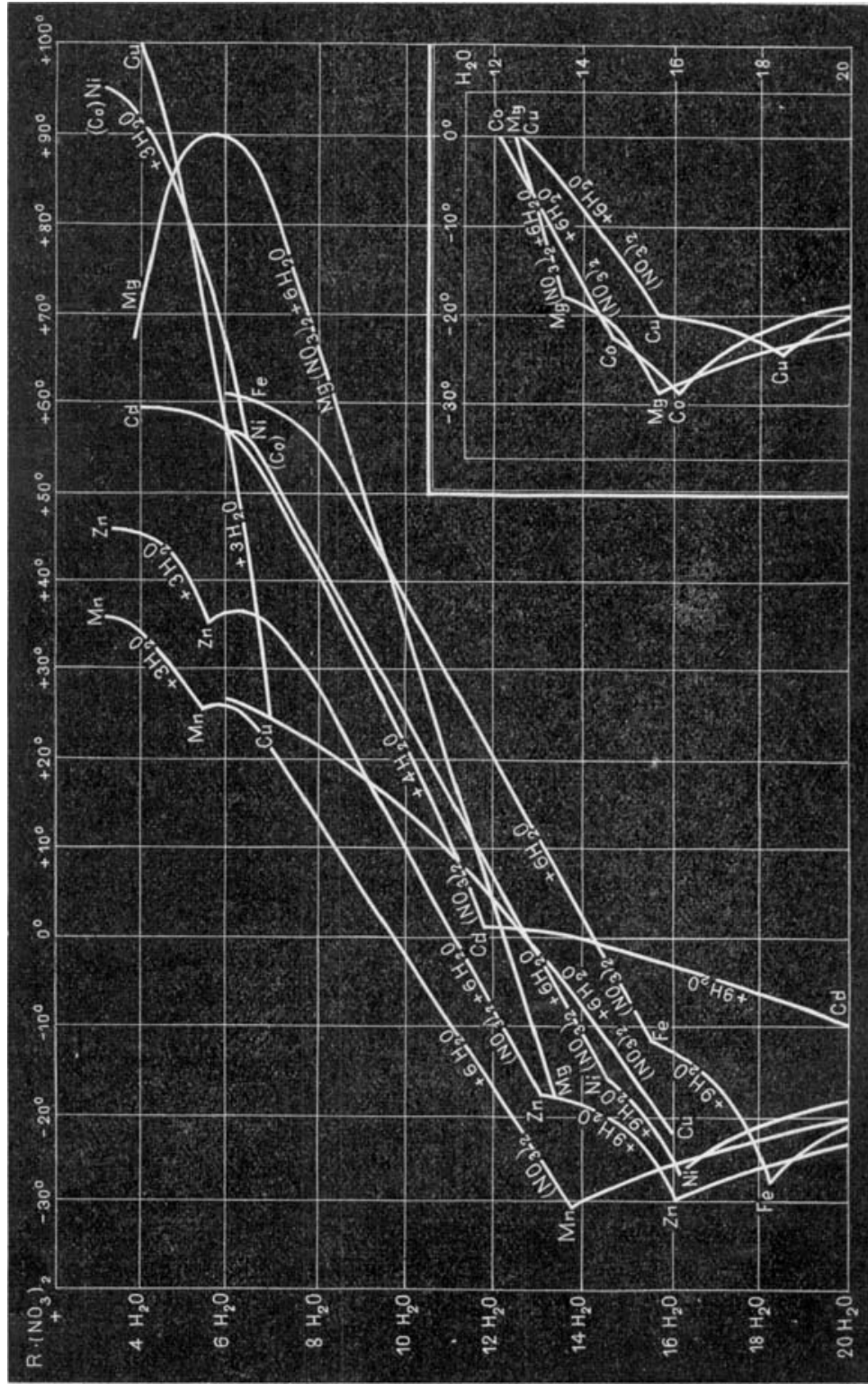

stabe zur Eisabscheidung gebracht, und dass nun bei vorsichtiger Erwärmung die Temperaturen beobachtet wurden, bei welchen sich das Eis gerade wieder auflöste. Es wurden folgende Daten gefunden: 


\begin{tabular}{c|c|c}
\hline \hline Temperatur & $\begin{array}{c}\text { Procente der Lösung } \\
\text { an } \mathrm{Mg}\left(\mathrm{NO}_{3}\right)_{2}\end{array}$ & $\begin{array}{c}\text { Moleküle Wasser auf } \\
1 \text { Mol. } \mathrm{Mg}\left(\mathrm{NO}_{3}\right)_{2}\end{array}$ \\
\hline-170 & 25.92 & 23.56 \\
$-22^{0}$ & 29.13 & 20.05 \\
$-26^{0}$ & 32.60 & 17.04
\end{tabular}

Im kryohydratischen Punkt bei $-29^{\circ}$ stösst die Gefrierpunktgcurve mit der Curve des wasserreichsten Hydrates zusammen; von $-29^{\circ}$ bis $-18^{\circ}$ ist das letztere, von da an das Hexahydrat stabil.

II. Zinknitrat.

Das Zinknitrat krystallisirt bei Zimmertemperatur bekanntlich mit 6 Mol. Wasser. Ausser diesem Hydrat sind aber noch 2 Modificationen existenzfähig, eine mit $3 \mathrm{Mol}$. Wasser oberhalb $36^{\circ}$ und eine wasserreichere mit 9 Mol. Wasser unter $-18^{\circ}$.

Die Löslichkeitsbestimmungen der drei Hydrate ergaben:

\begin{tabular}{|c|c|c|c|}
\hline Hydrat & Temperatur & $\begin{array}{c}\text { Procente } \\
\text { der Lösung an } \\
\mathrm{Zn}\left(\mathrm{NO}_{3}\right)_{2}\end{array}$ & $\begin{array}{l}\text { Moleküle Wasser } \\
\text { anf } \\
1 \mathrm{Mol} . \mathrm{Zn}\left(\mathrm{NO}_{3}\right)_{2}\end{array}$ \\
\hline $\mathrm{Zn}\left(\mathrm{NO}_{3}\right)_{2}+6 \mathrm{H}_{2} \mathrm{O}$ & 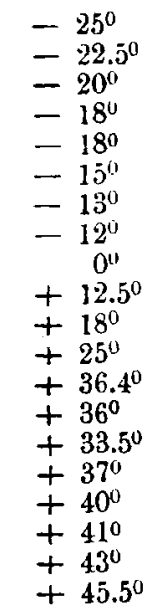 & $\begin{array}{l}40.12 \\
40.75 \\
42.03 \\
43.59 \\
44.63 \\
45.26 \\
45.51 \\
45.75 \\
48.66 \\
52.00 \\
53.50 \\
55.90 \\
63.63 \\
64.73 \\
65.83 \\
66.38 \\
67.42 \\
68.21 \\
69.26 \\
77.77\end{array}$ & $\begin{array}{l}15.70 \\
15.30 \\
14.51 \\
13.62 \\
13.05 \\
12.73 \\
12.60 \\
12.48 \\
11.10 \\
9.71 \\
9.14 \\
8.30 \\
6.00 \text { Schmp. } \\
5.73 \\
5.46 \\
5.33 \\
5.08 \\
4.90 \\
4.67 \\
3.00 \text { Schmp. }\end{array}$ \\
\hline
\end{tabular}

Für die Gefrierpunktecurve wurden folgende Punkte bestimmt:

\begin{tabular}{c|c|c}
\hline Temperatur & $\begin{array}{c}\text { Procente der Lösung } \\
\text { an } \mathrm{Zn}\left(\mathrm{NO}_{3}\right)_{2}\end{array}$ & $\begin{array}{c}\text { Moleküle Wasser auf } \\
\text { 1 Mol. } \mathrm{Zn}\left(\mathrm{NO}_{3}\right)_{2}\end{array}$ \\
\hline$-16^{\circ}$ & 30.00 & 24.55 \\
$-23.5^{\circ}$ & 35.00 & 19.54 \\
$-29.5^{\circ}$ & 39.65 & 16.02 \\
$-34^{\circ}$ & 42.85 & 14.03
\end{tabular}


Die Gefrierpunktscurve kann also über den kryobydratischen Punkt hinaus verfolgt werden. Bei $-29^{\circ}$ stösst sie auf die Curve des wasserreichsten Hydrates, welche ihrerseits bei $-17.5^{\circ}$ von der Curve des Hexahydrates geschuitten wird. Von $-17.5^{\circ}$ an aufwärts ist das Hexahydrat stabil, seine Curve zeigt vom Schmp. (36.40) aus eine rückläufige Fortsetzung uud schneidet auf dieser noch oberhalb $34^{\circ}$ die Curve des Trihydrates; diese wiederum findet bei $45.5^{\circ}$, dem Schmelzpunkt des Trihydrates, ihren Abschluss.

Weder durch Abdunsten im Vacuum über Schwefelsäure, noch bei Temperaturen über $45.5^{\circ}$ liess sich ein noch wasserärmeres Salz gewinnen; alle Versuche nach dieser Ricbtung scheiterten an dem gleichzeitigev Entweichen von Salpetersäure.

\section{Manganuitrat.}

Das bei Zimmertemperatur erhaitene Mangannitrat enthält 6 Mol. Wasser, es schmilzt bei $25.5^{\circ}$; ausserdem kaun man ein Trihydrat gewinuen, welches bei $35.5^{0}$ schmilzt.

Für die Festlegung der Löslichkeitscurven beider Hydrate wurden folgende Betimmungen ausgeführt:

\begin{tabular}{|c|c|c|c|}
\hline Hrdrat & Temperatur & $\begin{array}{c}\text { Procente } \\
\text { der Lösung an } \\
\mathrm{Mn}\left(\mathrm{NO}_{3}\right)_{2}\end{array}$ & $\begin{array}{l}\text { Moleküle Wasser } \\
1 \mathrm{Mol} . \mathrm{Mn}\left(\mathrm{NO}_{3}\right)_{2}\end{array}$ \\
\hline $\mathrm{Mn}\left(\mathrm{NO}_{3}\right)_{2}+3 \mathrm{H}_{2} \mathrm{O}$ & 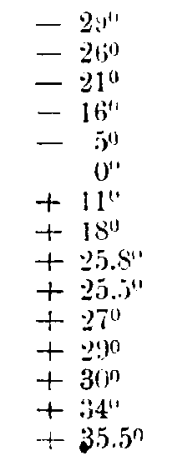 & $\begin{array}{l}4.2 .2 ! \\
43.15 \\
44.30 \\
45.59 \\
48.88 \\
50.49 \\
54.50 \\
57.33 \\
62.37 \\
65.99 \\
65.66 \\
66.9 ! \\
67.39 \\
71.31 \\
76.89\end{array}$ & $\begin{array}{l}13.57 \\
13.11 \\
12.50 \\
11.91 \\
10.40 \\
9.75 \\
8.01 \\
7.40 \\
6.10 \text { Schmp. } \\
5.35 \\
5.21 \\
4.90 \\
4.82 \\
4.01 \\
3.00 \text { Schmp. }\end{array}$ \\
\hline
\end{tabular}

Für die Gefrierpunktscurve wurden folgende Werthe gefunden:

\begin{tabular}{c|c|c}
\hline \hline Temperatur & $\begin{array}{c}\text { Procente der Lösung } \\
\text { an } \mathrm{Mn}\left(\mathrm{NO}_{3}\right)_{2}\end{array}$ & $\begin{array}{c}\text { Moleküle Wasser auf } \\
\text { 1 Mol. } \mathbf{M n}\left(\mathrm{NO}_{3}\right)_{\mathbf{2}}\end{array}$ \\
\hline$-15.5^{\prime \prime}$ & 29.30 & 24.01 \\
$-20^{\prime \prime}$ & 32.98 & 20.20 \\
$-30^{\circ}$ & 41.70 & 13.91
\end{tabular}


Aus der Curvenzeichnung ersieht man, dass sich die Curven des Tri- und Hexa-Hydrates bei etwa $25^{\circ}$ auf dem rückläutigen Theil der Curve des letzteren scbneiden, über dieser Temperatur ist das Tribydrat, unter derselben das Hexahydrat stabil. Der Schnittpunkt der Eiscurve und der Curve des Hexabydrates liegt bei $-30^{\circ}$. Der skryohydratische Punkt wurde andererseits aber bei $-36^{\circ}$ gefunden; es ist daher möglich, dass die Eiscurie hier von der Curve eines wasserreicheren Hydrates - wahrscheinlich analog den entsprechenden Hydraten der anderen Nitrate mit 9 Mol. Wasser - geschnitten wird.

\section{Eisennitrat.}

Das bei Zimmertemperatur erbïltliche Eisennitrat krystallisirt mit 6 Mol. Wasser. Das Salz ist ausserordentlich zersetzlich and hält sich bei Zimmertemperatur nicht länger als $1-2$ Tage. Bei $0^{0}$ ist es wesentlich haltbarer, doch tritt auch hier schliesslicb Oxydation ein. Der Schmelzpunkt des Salzes liegt bei $60.5^{\circ}$, das geschmolzene Salz zersetzt sich, nur wenig über den Schmelzpunkt erhitzt, sofort unter Gasentwickelung; beim Stehen über Schwefelsäure tritt in wenigen Minuten die Zersetzung ein. An die Gewinnung eines wasserärmeren Hydrates ist also nicht zu denken.

Ausser dem Hexabydrat ist analog den anderen Nitraten bei tiefen Temperaturen eine wasserreichere Modification, vermuthlich mit 9 Mol. Wasser, zu erbalten. Was die Löslichkeit des Eisennitrats in Wasser betrifft, so ist dieselbe für das Hexahydrat bereits von Ordway ${ }^{1}$ ) festgestellt wordes. dessen Werthe sich von den meinigen nur wenig unterscheiden.

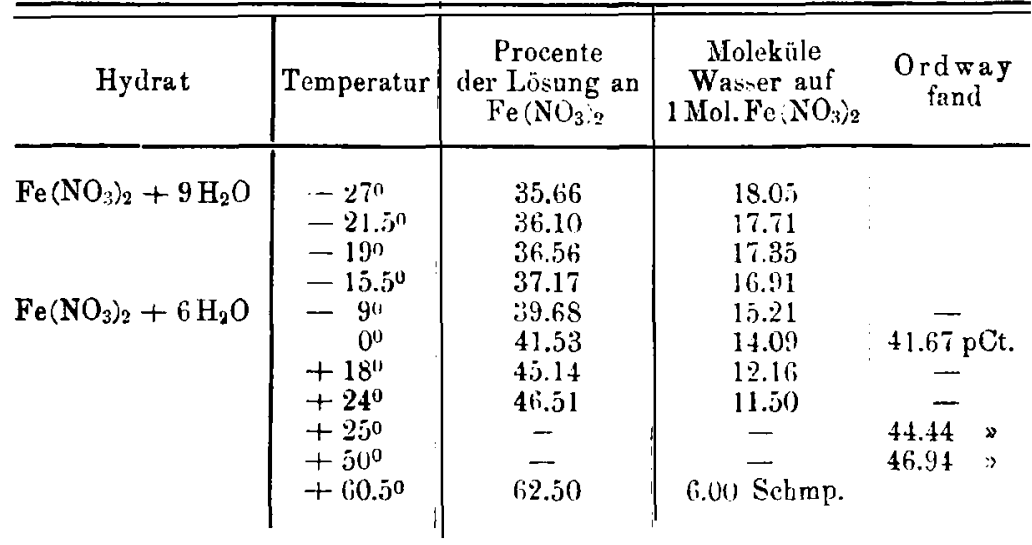

Die Curve des Hydrates mit 9 Molekülen Wasser schneidet also die Curve des Hexahydrates bei etwa $-12^{n}$, sie endigt im kryo-

1) Sill [2] 40, 325. 
hydratischen Punkt bei $-28^{\circ}$; hier stösst sie auf die Eiscurve, für welche die Bestimmungen folgende Werthe ergaben.

\begin{tabular}{c|c|c}
\hline \hline Temperatur & $\begin{array}{c}\text { Procente der Lösung } \\
\text { an } \mathrm{Fe}\left(\mathrm{NO}_{3}\right)_{2}\end{array}$ & $\begin{array}{c}\text { Moleküle Wasser auf } \\
1 \mathrm{Mol} . \mathrm{Fe}\left(\mathrm{NO}_{3}\right)_{2}\end{array}$ \\
\hline$-14.5^{\circ}$ & 29.76 & 23.62 \\
$-190^{\circ}$ & 32.36 & 20.91 \\
-210 & 33.33 & 20.01
\end{tabular}

\section{Kobaltnitrat.}

Das gewöhnliche Kobaltnitrat hat bekanntlich die Zusammensetzung $\mathrm{Co}\left(\mathrm{NO}_{3}\right)_{2}+6 \mathrm{H}_{2} \mathrm{O}$. Ausserdem kann man ein Hydrat mit 3 und eins mit 9 Molekülen Wasser herstellen.

Bei den Löslichkeitsbestimmungen dieser drei Modificationen wurden folgende Werthe gefunden.

\begin{tabular}{|c|c|c|c|}
\hline Hydrat & Temperatur & $\begin{array}{l}\text { Procente der } \mathrm{Lö-} \\
\text { sung an } \mathrm{Co}\left(\mathrm{NO}_{3}\right)_{2}\end{array}$ & $\begin{array}{c}\text { Moleküle Wasser auf } \\
\text { I Mol. } \mathrm{Co}\left(\mathrm{NO}_{3}\right)_{2}\end{array}$ \\
\hline \multirow[t]{3}{*}{$\mathrm{Co}\left(\mathrm{NO}_{3}\right)_{2}+9 \mathrm{H}_{2} \mathrm{O}$} & $-26^{\circ}$ & 39.45 & 15.62 \\
\hline & $-23.5^{n}$ & 40.40 & 15.02 \\
\hline & $-20.5^{\circ}$ & 42.77 & 13.61 \\
\hline \multirow{7}{*}{$\mathrm{Co}\left(\mathrm{NO}_{3} \cdot 2+13 \mathrm{H}_{2} \mathrm{O}\right.$} & $-21^{0}$ & $41.5 j$ & 14.32 \\
\hline & $-10^{0}$ & 43.69 & 13.11 \\
\hline & -40 & 44.85 & 12.51 \\
\hline & $0^{0}$ & 45.66 & 12.11 \\
\hline & $+18^{\circ}$ & $49.7 \mathfrak{\Xi}$ & 10.30 \\
\hline & $+41^{0}$ & 55.96 & 8.01 \\
\hline & $+56^{11}$ & $62.8 \AA$ & 6.00 Schmp. \\
\hline \multirow{4}{*}{$\mathrm{Co}\left(\mathrm{NO}_{3}\right)_{2}+3 \mathrm{H}_{2} \mathrm{O}$} & $+55^{0}$ & 61.74 & 6.31 \\
\hline & +620 & 62.88 & 6.01 \\
\hline & $+70^{0}$ & 64.89 & 5.51 \\
\hline & $\begin{array}{l}+84^{0} \\
+91^{0}\end{array}$ & $\begin{array}{l}68.84 \\
77.21\end{array}$ & $\begin{array}{l}4.61 \\
3.00 \text { Sehmp. }\end{array}$ \\
\hline & & & \\
\hline
\end{tabular}

Das Trihydrat schmilzt bei $91^{\circ}$, von hier an bis $55^{\circ}$, wo die Curve des Trihydrates die des Hexabydrates schneidet, ist es beständig. Da das Hexahydrat oberhalb des Schnittpunktes erst bei $56^{0}$ schmilzt, so stellt dasselbe im geschmolzenen Zustand eine übersättigte Lösung vor: wendet man daher zur Bestimmung des Schmelzpunktes grössere Mengen an, so tritt eine Umwandlung in das bei dieser Temperatur stabilere Tribydrat ein, und man erhält bei $56^{\circ}$ eine gesättigte Lösung dieses letzteren, welches sich als Bodensalz erst bei $62^{\circ}$ auf lōst.

Die Curve des Hexahydrates endigt bei etwa $-22^{0}$; hier zweigt sich die Curve des Hydrates mit 9 Molekülen Wasser ab, welche ihrerseits im kryohydratischen Punkt bei $-29^{\circ}$ ihren Abschluss 
Gindet. Für die Gefrierpunktscurve, welche bier einsetzt, wurden folgende Werthe gefunden.

\begin{tabular}{|c|c|c|}
\hline Temperatur & $\begin{array}{l}\text { Procente der Lösung } \\
\text { an } \mathrm{Co}\left(\mathrm{NO}_{3}\right)_{2}\end{array}$ & $\begin{array}{c}\text { Moleküle Wasser auf } \\
1 \text { Mol. Co } \text { NO:; }^{2}\end{array}$ \\
\hline $\begin{array}{l}-14^{\prime \prime} \\
-15^{0} \\
-22^{\prime \prime}\end{array}$ & $\begin{array}{l}24.4 ! \\
3.8 .5 \\
31.35\end{array}$ & $\begin{array}{l}24.32 \\
20.80 \\
17.81\end{array}$ \\
\hline
\end{tabular}

VI. Nickelnitrat.

Die grosse $\Lambda$ ehulichkeit zwischen Kobalt und Nickel zeigt sich auch in dem Verbalten ihrer Nitrate. Auch das Nickelnitrat erscheint in 3 Moditicationen - mit 3,6 und ! Molekülen Wasser - und zeigt in seiner Löslichkeit nur wenig Abweichung vom Kobaltnitrat. Der Schmelzpunkt des Hexahydrates liegt bei $56.7^{\prime \prime}$, das Trihydrat schmilzt bei $95^{\circ}$, also $4^{n}$ höher, als das entsprecbende Kobaltsalz.

Die Löslichkeitsbestimmungen ergaben folgende Werthe:

\begin{tabular}{|c|c|c|c|}
\hline Hydrat & Temperatur & $\begin{array}{l}\text { Procente der } \mathrm{Loi-} \\
\text { sung an } \mathrm{Ni}\left(\mathrm{NO}_{3}\right)_{2}\end{array}$ & $\begin{array}{c}\text { Moleküle Wasser auf } \\
1 \text { Mol. } \mathrm{Ni}\left(\mathrm{NO}_{3}\right)_{2}\end{array}$ \\
\hline \multirow{3}{*}{$\mathrm{Ni}\left(\mathrm{NO}_{3}\right)_{2}+! ! \mathrm{H}_{2} \mathrm{O}$} & $-23^{\prime \prime}$ & $3 ! 1.012$ & 1.j.x \\
\hline & $-l^{\prime \prime}$ & $3 ! 1.48$ & 15.56 \\
\hline & $-10.5^{i 3}$ & 44.13 & $1-2.8 .1$ \\
\hline \multirow{8}{*}{$\mathrm{Ni}\left(\mathrm{NO}_{3}\right)_{2}+6 \mathrm{H}_{2} \mathrm{O}$} & $-2 ! 1$ & 3.1 .14 & 15.27 \\
\hline & $-12.5^{14}$ & $41.5 ! 1$ & 14.26 \\
\hline & -111 & 42.11 & $1: 3.96$ \\
\hline & - in & $4:(x)$ & 13.419 \\
\hline & ()$^{n}$ & $44.3 \%$ & 12.75 \\
\hline & +211 & $4 ! .616$ & 10.54 \\
\hline & $+41^{11}$ & 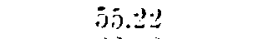 & 5.23 \\
\hline & $+56.7^{0}$ & 62.66 & li.gul Sehmp. \\
\hline \multirow[t]{6}{*}{$\mathrm{Ni}\left(\mathrm{NO}_{2}\right)_{2}+3 \mathrm{H}_{2} \mathrm{H}$} & $+58^{\prime \prime}$ & (i1.6)1 & 6.33 \\
\hline & $+600^{0}$ & (i1,.!!! & $6.2: 3$ \\
\hline & $+64 "$ & $(i \underline{-71 i}$ & $6.0 ;$ \\
\hline & $+\pi(n$ & (ii3.135 & 5.72 \\
\hline & $\begin{array}{l}+! 10 \\
+450\end{array}$ & 70.16 & 4.32 \\
\hline & $+1,0^{\circ}$ & 6.12 & $\therefore .(1)$ \\
\hline
\end{tabular}

Der Schnittpunkt der Curven des Trihydrates und Hexahydrates liegt wie beim Kobaltnitrat bei etwa $55^{\circ}$. In der Näbe dieses Punktes zeigen Kobalt- und Nickel-Nitrat fast die gleiche Löslichkeit, während die Curven der beiden Hexahydrate und der beiden Trihydrate allmählich mit zunehmender Entfernung von diesem Punkt aus einander geben.

Die Curre des Hexahydrates wird bei $-16^{\circ}$ von der Curve des Hydrates mit "Molekülen Wasser greschuitten. Diese endigt im kryohydratischen Punkt bei $-2 \tau^{0}$. 
Für die Gefrierpunktseurve ergaben die Bestimmungen folgende Werthe:

\begin{tabular}{|c|c|c|}
\hline 'T'emperatur & $\begin{array}{c}\text { Procente der lösung } \\
\text { an } \mathrm{Ni}\left(\mathrm{NO}_{3}\right)_{2}\end{array}$ & $\begin{array}{c}\text { Moleküle Wasser auf } \\
\text { l Mol. } \mathrm{Ni}\left(\mathrm{NO}_{3}\right)_{2}\end{array}$ \\
\hline $\begin{array}{l}-15^{11} \\
-18.5^{11} \\
--13^{41}\end{array}$ & $\begin{array}{l}30.63 \\
3356 \\
37.2 ! !\end{array}$ & $\begin{array}{l}29.00 \\
20.18 \\
17.01\end{array}$ \\
\hline
\end{tabular}

Die Eiscurve länft dicht neben der Eiscurse des Kobaltnitrates her.

\section{Kupfernitrat.}

Das Kupfernitrat ist in zwei verschiedenen Hydratformen bekannt, mit $t$ and mit 3 Mnlekülen Wasser. Ausserdem existirt aber eine wasserreichere Modification mit ? Molekülen Wasser. Das Hexahydrat schmilzt bei $26.4^{0}$ und zerfällt wie das entsprechende Kobaltund Nickel-Salz bei der Schmelztemperatur in das wasserärmere Tribydrat und dessen gesättigte Lösung. Der Schmelzpunkt des Trihydrates liegt bei $114.5^{\circ}$.

Die Löslichkeitsbestimmungen für die drei Hydrate ergaben:

\begin{tabular}{|c|c|c|c|}
\hline Hydrat & Temperatur & $\begin{array}{l}\text { Procente der Lö- } \\
\text { sung an } \mathrm{Cu}\left(\mathrm{N}\left(U_{3}\right), 2\right.\end{array}$ & $\begin{array}{c}\text { Moleküle Wasser auf } \\
1 \text { Mol. ('u(NO): }\end{array}$ \\
\hline \multirow[t]{3}{*}{$\mathrm{Cu}\left(\mathrm{NO}_{32}\right)_{2}+! \mathrm{H}_{2} \mathrm{O}$} & $-23^{\prime \prime}$ & 3 foi & 18.45 \\
\hline & -210 & 37.38 & $173 ! 1$ \\
\hline & $-20^{\prime \prime}$ & $40.9 \cdot 3$ & 15.04 \\
\hline \multirow[t]{6}{*}{$\mathrm{Cu}(\mathrm{NO})_{2}+\left(\mathrm{H}_{2} \mathrm{O}\right.$} & $-21^{\prime \prime}$ & 39.82 & 15.94 \\
\hline & -100 & 42.015 & 14.34 \\
\hline & & 45.160 & 12.7i; \\
\hline & $+1(1)$ & 48.79 & 10.918 \\
\hline & $+2\left(\mu^{\prime \prime}\right.$ & 5.58 & 5.82 \\
\hline & $+26.4^{0}$ & $(3: 3.3 !)$ & li (00) Schmp. \\
\hline \multirow[t]{7}{*}{$\mathrm{Cu}\left(\mathrm{NO}_{3}\right)_{2}+3 \mathrm{H}_{0} \mathrm{O}$} & +2.511 & 6ic.(1) & 6.94 \\
\hline & $+: 30^{\prime \prime}$ & (iio.t. & (; 82 \\
\hline & $\begin{array}{l}+40^{\prime \prime} \\
+50^{61}\end{array}$ & $(i) . j)$ & 6.52 \\
\hline & $\begin{array}{l}+5\left(0^{\prime \prime}\right. \\
+6 w^{\prime \prime}\end{array}$ & 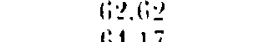 & 6.20 \\
\hline & $+71^{\prime \prime}$ & (15.7) & $5.4 y$ \\
\hline & $+\div(100$ & 67.51 & 5011 \\
\hline & $114.5^{01}$ & $77.3 !)$ & 3.(n) Sithp. \\
\hline
\end{tabular}

Die Gefrierpunktsbestimmungen ergaben:

\begin{tabular}{c|c|c}
\hline \hline Temperatur & $\begin{array}{c}\text { Procente der } \mathrm{Lösung} \\
\text { an } \mathrm{Cu}\left(\mathrm{NO}_{3}\right)_{2}\end{array}$ & $\begin{array}{c}\text { Moleküle Wasser auf } \\
1 \mathrm{Mol} . \mathrm{Cu}\left(\mathrm{NO}_{3}\right)_{2}\end{array}$ \\
\hline$-16^{\circ}$ & 30.93 & 23.26 \\
$-20^{\circ}$ & 34.29 & 19.96
\end{tabular}


Die Curve des Hexahydrates schneidet die Curce des Trihydrates bei efwa $24.5^{\circ}$ und die Curve des Hydrates mit 9 Molekülen Wasser bei $-20^{\circ}$. Bei $-24^{0}$, dem kryohydratischen Punkt, setzt die Grfrierpunktscurve ein.

\section{Cadmiumnitrat.}

Während sämmtliche. hier beschriebene Nitrate bei Zinmertemperatur mit 6 Molekülen Wasser krystallisiren, hat das gewöbnliche Cadmiumnitrat die Zusammensetzung $\mathrm{Cd}\left(\mathrm{NO}_{3}\right)_{2}+4 \mathrm{H}_{2} \mathrm{O}$. Es krystallisirt in strahlig zusammengehäuften Säulen und Nadeln, welcbe bei $59.5^{\circ}$ schmelzen. Bei tiefen Temperaturen setzen sie sich langsam in wasserreichere Krystalle um von der Formel $\mathrm{Cd}\left(\mathrm{NO}_{3}\right)_{2}$ $+9 \mathrm{H}, \mathrm{O}$. Diese Umsetzung geht wesentlich schwerer von statten, als bei den anderen Nitraten; am besten rerfährt man, wenu man die übersättigte Lösung auf etwa $-30^{0}$ abłühlt, unter plötzlicher Temperatursteigerung bis $-16^{0}$ tritt dann die Bilduog des Kryohydrates ein, ans welchem beim Aufthauen die wasserreichste Modification isolirt werden kann.

Für die bei den einzelnen Temperaturen gesättigten Lösungen wurde folgende Zusammensetzung gefunden.

\begin{tabular}{|c|c|c|c|}
\hline Hydrat & $\mathrm{T} \cdot \mathrm{mperatur}$ & $\begin{array}{l}\text { Procente der } \mathrm{Lo}- \\
\text { sung an } \mathrm{Cd}\left(\mathrm{NO}_{3}\right)_{2}\end{array}$ & $\begin{array}{c}\text { Moleküle Wasser auf } \\
1 \text { Mol. Ca No }\end{array}$ \\
\hline $\begin{array}{l}\mathrm{Cd}\left(\mathrm{NO}_{3,2}+4 \mathrm{H}_{2} \mathrm{O}\right. \\
\mathrm{Cd}\left(\mathrm{NO}_{42}+4 \mathrm{H}_{2} \mathrm{O}\right.\end{array}$ & $\begin{array}{c}-13^{\prime \prime} \\
-\quad 1^{\prime \prime} \\
+\quad 1^{\prime \prime} \\
9^{\prime \prime} \\
15^{\prime \prime} \\
30^{\prime \prime} \\
40^{\prime \prime} \\
59.5^{\prime \prime}\end{array}$ & $\begin{array}{l}37.37 \\
47.39 \\
52.73 \\
52.31 \\
55.60 \\
38.40 \\
61.4 .2 \\
76.54\end{array}$ & $\begin{array}{l}21.98 \\
14.5 ! 1 \\
11.74 \\
11.96 \\
10.34 \\
11.34 \\
8.24 \\
4.00 \text { Schmn. }\end{array}$ \\
\hline
\end{tabular}

Die Gefrierpunktscurve wurde durch folgende Bestimmungen ermittelt:

\begin{tabular}{|c|c|c|}
\hline Temperatur & $\begin{array}{c}\text { Procente der Lösung } \\
\text { an } \mathrm{Cd}\left(\mathrm{NO}_{3}\right)_{2}\end{array}$ & $\begin{array}{c}\text { Moleküle Wasser anf } \\
1 \text { Nol. } \mathrm{Cd}(\mathrm{NO}:)_{2}\end{array}$ \\
\hline $\begin{array}{l}-1.3^{\prime \prime} \\
-13^{11} \\
-14.5^{\prime \prime}\end{array}$ & $\begin{array}{l}30.68 \\
3.5 .62 \\
35.91\end{array}$ & $\begin{array}{l}29.64 \\
23: .71 \\
23.41\end{array}$ \\
\hline
\end{tabular}

Die Curven der beiden Hydrate schneiden sich also bei $+1^{0}$, die Curve des Hydrates mit 9 Molekülen Wasser fiallt ron hier aus steil ab und endigt bei $-16^{\circ}$ im kryohydratiscten Punkt. wo sich die Gefrierpunktscurve ansetzt. 
Die Curve des Tetrahydrates hat zweifellos eine rückläufige 'Fortsetzung, denn man kann Lösungen von dem Hydrat berstellen, welche weniger als 4 Moleküle Wasser enthalten, dieselben sind aber so viscos, dass es fast unnöglich erscheint, Bestimmungen mit einiger Sicherheit auszuführen. Man kann jedoch die Curve soweit verfolgen, dass die gesättigte Lösung weniger als 2 Moleküle Wasser enthält.

Jenseits des Schmelzpunktes des Tetrahydrats krystallisirt noch ein wasserärmeres Hydrat mit 2 Molekülen Wasser, dessen Löslichkeit jedoch nicht weiter verfolgt wurde. Der Schmelzpunkt desselben liegt jenseits $130^{\circ}$, wo bereits Zersetzung eintritt.

Die vorstehend beschriebenen Metallnitrate sind von jeher zu einer Gruppe zusammengefisst worden; mit Ausnabme des Cadmiumsalzes kennt man sie in der Modification mit 6 Molekülen Krystallwasser; sie sind daher zur Vergleichung der Löslichkeit ebenso geeignet wie die Gruppe der Vitriole. Wie bei diesen') lässt sich die Vergleichung auch auf das Cadmiumsalz ausdehnen, sobald man die Beobachtung bei niedrigen Temperaturen vornimmt; die Modification mit 9 Molekülen Wasser ist allen erwähnten Nitraten gemeinsam.

Vergleicht man die Löslichkeit der Nitrate an den kryohydratischen Punkten, so findet man folgende Reihe für die Hydrate mit 9 Molekülen Wasser, in welcher das Cadmiumsalz als das am wenigsten lösliche erscheint. Die Lösung enthält auf 1 Molekül wasserfreies Salz:

\begin{tabular}{|c|c|c|}
\hline Nitrate & Moleküle Wasser & $\begin{array}{c}\text { Kryohydratische } \\
\text { Punkte }\end{array}$ \\
\hline $\begin{array}{l}\mathrm{Cd}\left(\mathrm{NO}_{3}\right)_{2} \\
\mathrm{Cu}\left(\mathrm{NO}_{3}\right)_{2} \\
\mathrm{Fe}(\mathrm{NO})_{2} \\
\mathrm{Ni}(\mathrm{NO})_{2} \\
\mathrm{Zn}(\mathrm{NO})_{2} \\
\mathrm{Co}\left(\mathrm{NO}_{3}\right)_{2} \\
\mathrm{Mg}(\mathrm{NO})_{2} \\
\mathrm{Mu}(\mathrm{NO})_{2}\end{array}$ & $\begin{array}{l}23.1 \\
18.5 \\
15.1 \\
16.2 \\
11.1 \\
16.11 \\
15.7 \\
12-14\end{array}$ & 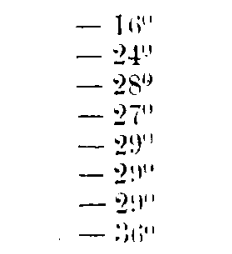 \\
\hline
\end{tabular}

Die Löslichkeit wächst also in nmgekehrter Richtung wie die Temperatur der kryobydratischen Punkte.

Charlottenburg, im December 1 sis.

1) Mylins nud Funk, diese Beriehte 30, $8: 4 \mathrm{ff}$. 